\title{
Astrophysical neutrinos and atmospheric leptons
}

\section{T.K. Gaisser ${ }^{\mathrm{a}}$}

Bartol Research Institute and Department of Physics and Astronomy, University of Delaware, Newark, DE 19716, USA

\begin{abstract}
IceCube measurements of the neutrino flux from $\mathrm{TeV}$ to $\mathrm{PeV}$ show the signal of astrophysical neutrinos standing out at high energy well above the steeply falling foreground of atmospheric neutrinos. The astrophysical signal appears both in measurements of neutrino-induced muons and in the starting event sample, which responds preferentially to electron and tau neutrinos, but which also includes muon neutrinos. Searches for point sources of astrophysical neutrinos have, however, not yet identified a single source or class of sources for the astrophysical component. Some constraints on astrophysical sources implied by the current observations will be described in this talk. Uncertainties in the fluxes of atmospheric leptons resulting from an incomplete knowledge of the primary cosmic-ray spectrum and from a limited understanding of meson production, including charm will also be reviewed. The ultimate goal is to improve the understanding of the astrophysical spectrum in the transition to lower energy where atmospheric neutrinos dominate.
\end{abstract}

The main aspects of this presentation will be included in the author's Review Talk at the end of the Symposium.

\footnotetext{
a e-mail: gaisser@bartol.udel.edu
} 